\title{
On the Deployment of Moving Networks in Ultra-dense Urban Scenarios
}

\author{
Invited Paper \\ Yutao Sui*, Ismail Guvenc ${ }^{\dagger}$, and Tommy Svensson* \\ ${ }^{*}$ Dept. Signals and Systems, Chalmers University of Technology, Sweden. Email: \{yutao.sui, tommy.svensson\}@chalmers.se \\ ${ }^{\dagger}$ Electrical \& Computer Engineering, Florida International University, Miami, FL, USA. Email: iguvenc@ @iu.edu
}

\begin{abstract}
In future mobile communication systems, more users will rely on mobile data services while riding public transportation vehicles for either working or entertainment. To serve these vehicular users effectively, the deployment of moving base stations on public transportation vehicles is considered as one of the most promising solutions. Each public transportation vehicle forms a moving network (MN) inside the vehicle to serve the users on board. In this paper, we study the deployment of MNs in an ultra-dense urban scenario, and we identify that one of the key challenges is the inter-cell interference, which is worsen by the street canyon effects. In order to address this problem, we employ and compare various solutions to enhance the performance of MNs. We show that by using MNs that have advanced multi-antenna systems, the quality of service at the vehicular users is noticeably improved without obvious influence on the performance of regular outdoor users.
\end{abstract}

\section{INTRODUCTION}

The expectation of ubiquitous mobile data connectivity is increasing as more people rely on Internet services in their daily lives. A 1000-fold increase of wireless data traffic is expected within the next decade [1]. Both the industry and academia are working together, e.g., METIS and $5 \mathrm{GNOW}^{1}$, to meet the capacity demand of the 5th generation mobile communication systems [2], [3]. Due to the high penetration of smartphones, tablets, and ultrabooks, more users rely on wireless connections while riding public transportation vehicles. They are expecting similar availability of mobile data services while they are traveling or commuting as they are sitting still. Therefore, this study targets a special user group, i.e., vehicular user equipment (VUE) devices in well isolated public transportation vehicles.

Several studies raise the issue of how to service these VUEs efficiently [4]-[7]. One of the most promising solutions is to deploy moving relay nodes (MRNs) on the vehicles, which circumvent the vehicular penetration loss (VPL) and improve the quality-of-service (QoS) at the VUEs. VPL can be as high as $25 \mathrm{~dB}$ in a minivan at the frequency of $2.4 \mathrm{GHz}$ [8], and higher VPLs are foreseeable in well isolated vehicles and at higher frequency bands allocated to future mobile communication systems. Earlier studies in [5], [9], [10] showed that in a noise limited system and system with limited interference, using half-duplex decode-and-forward MRNs could significantly

\footnotetext{
${ }^{1}$ METIS stands for Mobile and wireless communications Enablers for the Twenty-twenty Information Society; 5GNOW stands for 5th Generation NonOrthogonal Waveforms for Asynchronous Signaling.
}

improve the QoS at the VUEs. Furthermore, as a vehicle is less constrained by power and transceiver complexity, sophisticated multi-antenna solutions and more advanced signal processing techniques can be integrated to MRNs to further improve their performance. For example, in [6], it was showed that by using predicting antennas on top of high-speed vehicles, reliable channel state information (CSI) could be obtained at the MRNs to support advanced multi-antenna applications. Moreover, from a system point of view, the use of MRNs can significantly reduce the number of handover failures for high speed VUEs, and lower the signaling overhead for mobility management [7], [11]. However, to the best knowledge of the authors, the current study of using MRNs is either about system architectures or in very simplified scenarios. There is no detailed study about deploying MRNs in typical urban scenarios. New implementation challenges arise when we introduce a new type of node, i.e., the MRN, to the system, especially in a densely deployed urban scenario where the inter-cell interference becomes more complicated by the street canyon effects.

Contributions: to understand the practical challenges of deploying MNRs in an ultra-dense urban scenario, in this work, we extend our earlier work in [5], [9], [10] by considering a practical densely deployed heterogeneous and small cell networks (HetSNets) framework introduced by the EU 5G project METIS [3]. We assume each of the public transportation vehicles forms a moving network $(\mathrm{MN})$ to serve the VUEs on board. Depending on the availabilities of CSI at the receivers, we consider either the use of maximum ratio combining (MRC) or interference rejection combining (IRC) to enhance the reception of the backhaul links of MNs, and the use of almost blank subframes (ABSs) is explored to protect the access links of MNs. We show that by deploying MNs to the system, the throughput at the VUEs can be significantly improved, while the performance of regular outdoor users have no obvious degradation. Hence, the use of MNs constitutes a promising approach in future mobile communication systems to improve the experience of VUEs.

\section{SySTEM MODEL}

We consider the downlink of a densely deployed mobile network. To understand the benefits and challenges of deploying MNs in an ultra-dense urban HetSNet scenario, we align our study with the HetSNet framework defined by METIS [12], 


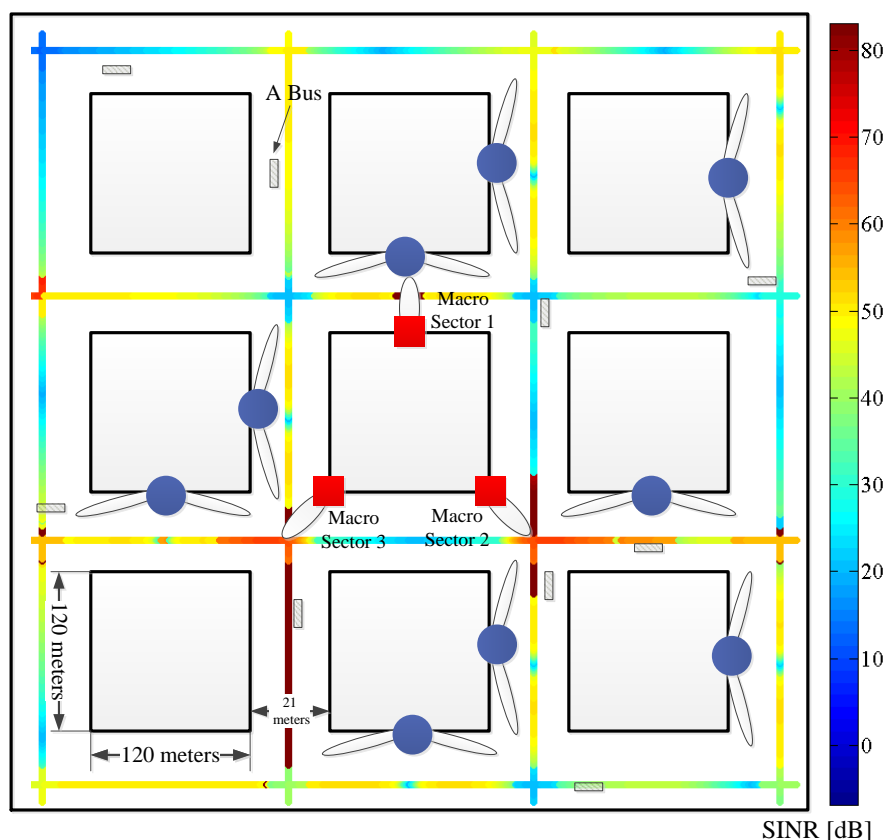

Macro Sector, 0, 120, and 240 degrees with respect to the north Micro station with 2 sectors, pointing to the main street with an angle of 20 degrees with respect to the closest wall Bus, 20 meters by 2 meters

Figure 1. Simplified Madrid grid model with a heterogeneous deployment of macro and micro BSs.

where a simplified deployment model is abstracted, as shown in Fig. 1. We use the calibration deployment parameters given in [12, Table 3.7], where each macro BS has three sectors for basic coverage, and each micro BS has two sectors for local coverage. Macro sectors operate at the $800 \mathrm{MHz}$ band with a $20 \mathrm{MHz}$ bandwidth while micro sectors transmit at $2.6 \mathrm{GHz}$ band with $80 \mathrm{MHz}$ bandwidth. We consider a general setup that each $\mathrm{MN}$ is deployed on a public transportation vehicle. The backhaul link, i.e., the link between the $\mathrm{MN}$ and the macro cells, is operating at $800 \mathrm{MHz}$, while the access link, i.e., the link between the MN and its VUEs, is using the same $2.6 \mathrm{GHz}$ frequency band as the micro cells. The MNs are assumed to work in a full duplex mode, as the backhaul and the access links are operating at different frequencies. An orthogonal frequency-division multiple access (OFDMA) based system is considered with the same parameters such as the sub-carrier spacing, physical resource block (PRB) size, and subframe length as the current Long-Term Evolution Advanced (LTEA) systems [13, Chp. 5 and 6]. We consider an intra-carrier aggregation of four carriers of $20 \mathrm{MHz}$ for the micro cells, since the current LTE-A system supports a maximum $20 \mathrm{MHz}$ bandwidth for one carrier.

\section{A. Interference scenario}

Unlike the traditional hexagonal deployment model, in ultradense urban scenarios, the interference situations are different, due to different propagation environments and the street canyon effect. Fig. 1 plots the signal-to-interference-plus-noise

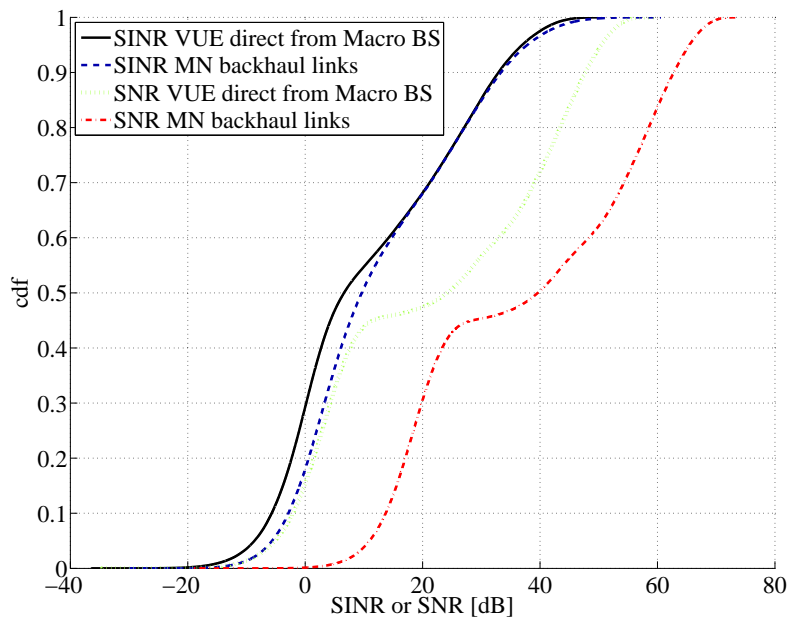

Figure 2. SNR and SINR plot at VUEs at 15dB VPL. The detailed parameters used for this plot are given in Section V.

ratio (SINR) that an outdoor user experiences on the street (the detailed parameters used for the SINR plot are given in Section V). As we can see from the figure, even when a user is close to a BS, e.g., between macro sector 2 and sector 3, it is not guaranteed a high SINR. As mentioned in Section I, one of the advantages of using MNs is to circumvent the VPL. However, in a densely deployed scenario, the inter-cell interference has a significant impact on the received SINR. Fig. 2 plots the cumulative distribution function (cdf) of the signal-to-noise ratio (SNR) and SINR at the VUEs and the backhaul links of MNs when the VPL is $15 \mathrm{~dB}$. Due to better propagation conditions, and that the MNs can eliminate the VPL, compared to the case when the VUEs are directly served by macro cells, the MNs can improve the SNR significantly. However, the SINR is not obviously improved due to severe inter-cell interference at the backhaul links of MNs.

The access links of MNs transmit at the same frequency bands as the micro cells, and therefore they suffer from the interference from the nearby micro cells. Although compared to the macro cells, the micro cells transmit at much lower power, the interference they cause to the access links of MNs is still significant since micro cells are densely deployed at street level. Moreover, it is common that the access links of an MN can be subject to the interference from several micro cells at the same time. Without proper interference management schemes, the access links of MNs cannot always accommodate the data from their backhaul links.

In this study, for the backhaul links of MNs, we consider the use of multi-antennas to improve the quality of the received signals. Depending on whether the CSI of the interfering sources can be observed or not, either MRC or IRC is implemented at the MN. Regarding the access links of MNs, we consider the use of ABSs at micro cells to reduce the impact of interference. These schemes are discussed in more detail in Section III and IV.

\section{B. Fairness among different user types}

Besides the study of interference management, to maintain the fairness between different kinds of users is also important. 
Our goal is to improve the performance of VUEs without degrading the experience of regular outdoor UEs. Therefore, in this study, we employ proportional fairness (PF) based scheduling to ensure a balance between system throughput and user fairness [14]. A full buffer traffic model is considered for all the users, as well as the same priorities for all the users. Moreover, each $\mathrm{MN}$ is treated as a super user that aggregates the traffic of all its VUEs. In a system without MNs, the throughput of $N_{\mathrm{u}}$ users is proportional fair [14], if the following objective function is maximized

$$
f_{1}=\sum_{u=1}^{N_{\mathrm{u}}} \log (s(u))
$$

where $\left\{s(u), u=1,2, \ldots, N_{\mathrm{u}}\right\}$, is the average throughput of the $u$ th user. However, when MNs are introduced into the system, the aggregated traffic of MNs for their VUEs needs to be taken into account, and the PF scheduler needs to be modified to ensure the fairness between VUEs and macro UEs. Let us introduce an additional $N_{\mathrm{m}}$ MNs into the system, and let $i_{v}$ denote the number of VUEs served by each MN where $v=1,2, \ldots, N_{\mathrm{m}}$. Then, the objective function of the PF scheduler is modified as

$$
f_{2}=\sum_{u=1}^{N_{\mathrm{u}}} \log (s(u))+\sum_{v=1}^{N_{\mathrm{m}}} \log \left(\frac{s(v)}{i_{v}}\right)
$$

where $\left\{s(v), v=1,2, \ldots, N_{\mathrm{m}}\right\}$ is the average throughput of the backhaul link of the $v$ th MN. The number of VUEs served by an $\mathrm{MN}$ is drawn uniformly as $i_{v} \sim \mathcal{U}(1,50)$ [12], where $\mathcal{U}$ denotes uniform distribution. The difference between (1) and (2) is that the traffic of the backhaul links of an MN is averaged by the number of its VUEs.

\section{ENHANCEMENT OF BACKHAUL LINKS OF MNS}

We consider using multi-antennas at the $\mathrm{MN}$ receivers to combat the inter-cell interference at the backhaul links. MNs on public transportation vehicles are less constrained by power and size compared to regular UEs. Hence, more antenna elements can be deployed and more advanced signal processing algorithms can be used at the receivers. A single transmit antenna is considered at each macro cell, and multiple antennas are assumed for the backhaul links receivers. Depending on the availability levels of CSI, various schemes can be used to improve the SINR at the receivers. We consider two cases, i.e., maximum ratio combining (MRC), and interference rejection combining (IRC).

\section{A. Maximum ratio combining}

MRC is a common way to coherently combine the desired signal from the receiver antennas to improve the received desired signal power. To use MRC, the receiver only needs to estimate the CSI of the desired signal at each of the antennas. An MRC receiver is optimal in terms of SNR, as it maximizes the output power of the desired signal [15]. We assume desired signal and interfering signals are uncorrelated. In a frequency flat fading case, at time $k$, the received signal is

$$
\mathbf{y}_{k}=\mathbf{h}_{k} x_{k}+\sum_{j=1}^{L} \mathbf{h}_{j, k} x_{j, k}+\mathbf{n}_{k},
$$

where $\mathbf{y}_{k}$ is a $p$ by 1 column vector that represents the signal at each receiver antenna, $x_{k}$ is the desired signal, and $x_{j, k}$ is the interfering signal from the $j$ th interferer. Here, $\mathbf{h}_{k}$ and $\mathbf{h}_{j, k}$ represent the desired and $j$ th interfering channel vectors, respectively, and $\mathbf{n}_{k}$ is the thermal noise. At the receiver, we apply a weight vector $\mathbf{w}_{k}$ at the signals received by each antenna at time $k$. Then the combined output signal is

$$
\mathbf{z}_{k}=\mathbf{w}_{k}^{\mathrm{H}} \mathbf{y}_{k}=\mathbf{w}_{k}^{\mathrm{H}} \mathbf{h}_{k} x_{k}+\sum_{j=1}^{L} \mathbf{w}_{k}^{\mathrm{H}} \mathbf{h}_{j, k} x_{j, k}+\mathbf{w}_{k}^{\mathrm{H}} \mathbf{n}_{k},
$$

where $(\cdot)^{\mathrm{H}}$ denotes the complex conjugate transpose. In an MRC receiver, the combining weights is given as $\mathbf{w}_{k}^{*}=\mathbf{h}_{k}$, which maximizes the desired signal power, and gives the instantaneous output SINR as [15]

$$
\gamma_{k}=\frac{\left|\mathbf{h}_{k}^{\mathrm{H}} \mathbf{h}_{k}\right|^{2}}{\left|\sum_{j=1}^{L} \mathbf{h}_{k}^{\mathrm{H}} \mathbf{h}_{j, k} x_{j, k}+\mathbf{h}_{k}^{\mathrm{H}} \mathbf{n}_{k}\right|^{2}} .
$$

From (4) we can see that at a given time, the output SNR is maximized; however, in general, the SINR is not maximized, due to the presence of the interfering signals. Nevertheless, this is the best one can achieve, if only the CSI of the desired signal can be obtained at the receiver side.

\section{$B$. Interference rejection combining}

If the CSI from the interferer can also be observed at each receiving antennas, we can use the multiple antennas at the receiver to suppress the interference, and maximize the output SINR. This scheme is usually referred to as IRC in the literature [16]. From (3), when assuming some arbitrary weight vector $\mathbf{w}_{k}$ at the received signal, we have the SINR as

$$
\gamma_{k}=\frac{\mathbf{w}_{k}^{\mathrm{H}} \mathbf{h}_{k} \mathbf{h}_{k}^{\mathrm{H}} \mathbf{w}_{k}}{\mathbf{w}_{k}^{\mathrm{H}}\left(\sum_{j=1}^{L} \mathbf{h}_{j, k}^{\mathrm{H}} \mathbf{h}_{j, k}+\mathbf{R}\right) \mathbf{w}_{k}},
$$

where $\mathbf{R}$ is the noise covariance for the receiver antennas, same for all $k$. Thus, if $\mathbf{h}_{j, k}$ is known, the optimal weight that maximize (5) is given by solving

$$
\mathbf{w}_{k}^{*}=\arg \max _{\mathbf{w}_{k}} \frac{\mathbf{w}_{k}^{\mathrm{H}} \mathbf{h}_{k} \mathbf{h}_{k}^{\mathrm{H}} \mathbf{w}_{k}}{\mathbf{w}_{k}^{\mathrm{H}}\left(\sum_{j=1}^{L} \mathbf{h}_{j, k}^{\mathrm{H}} \mathbf{h}_{j, k}+\mathbf{R}\right) \mathbf{w}_{k}} .
$$

Problem (6) is a generalized eigenvalue problem, and the optimal weight is given as [16]

$$
\mathbf{w}_{k}^{*}=\mathrm{EIG}_{\max }\left(\left(\sum_{j=1}^{L} \mathbf{h}_{j, k}^{\mathrm{H}} \mathbf{h}_{j, k}+\mathbf{R}\right)^{-1} \mathbf{h}_{k} \mathbf{h}_{k}^{\mathrm{H}}\right),
$$

where $\mathrm{EIG}_{\max }(\cdot)$ is the dominant eigenvector of a matrix. We assume $\mathbf{R}=\sigma_{\mathrm{n}}^{2} \mathrm{I}_{p \times p}$, where $\sigma_{\mathrm{n}}^{2}$ is the noise power at each antenna, and $\mathrm{I}_{p \times p}$ is the identity matrix of size $p$. Section $\mathrm{V}$ provides detailed system level evaluation results when MRC and IRC schemes are applied at the backhaul links of MNs. 


\section{INTERFERENCE COORDINATION FOR ACCESS LINKS}

The use of ABSs is considered at the micro cells to mitigate the interference that they cause to the access links of MNs. In LTE-A systems, ABSs are standardized to protect UEs that are subject to severe co-channel interference, as in the ABSs only some necessary signals are transmitted with low power [13, Chp. 31]. Compared to other interference coordination schemes, e.g., power control or coordinate scheduling, the advantage of using ABSs is that the micro cells do not need to acquire the CSI for all the VUEs they are interfering. As the coverage area of micro cells is relatively small, a public transportation vehicle can pass several micro cells in a short time. Since the typical time to update the ABSs setting is in the order of minutes [13, Chp. 31], it is reasonable to assume the same ABSs pattern is used by several micro cells in a given area to both minimize the signaling overhead for ABSs configurations, and reduce the overhead of measurement and feedback from the MNs to the network. Therefore, we assume the same ABSs configuration is used by all the micro cells in the area shown in Fig. 1. A reinforcement learning approach [17] is used to set the ABS patterns for the micro cells. The idea is to treat VUEs as primary users, and choose the ABS patterns from a pre-defined set iteratively to find the minimum number of ABSs that satisfies the average throughput requirements for the access link of the worst MN. This is because the goal here is to ensure the access links can accommodate the traffic from their backhaul links, rather than maximize the throughput of the access links.

We assume an ABS duty cycle of $40 \mathrm{~ms}$, i.e., the same ABS pattern is repeated very $40 \mathrm{~ms}$. For simplicity, we initialize the first subframe as ABS for all the micro cells, and then collect feedback from the MNs in the simulation area. If during the measurement time (set to one ABS duty cycle in this study), one or several MNs report insufficient number of ABSs, one more arbitrary subframe is added as ABS for more protection. We iterate this approach until the number of ABSs satisfies the average throughput of the access link of the worst MN. We assume MNs can buffer the data from their backhaul links. Hence, as long as the buffer is large enough, and the measurement time is reasonable short, there are no concerns about unnecessary package drops and excessive delays.

\section{Performance Evaluation}

The simulation of MNs is conducted in the area shown in Fig. 1, and the simulation parameters are set according to the calibration setups in [12, Table 3.7], and summarized in Table I. A full-buffer traffic model is assumed, and the PF scheduling described in Section II-B is used. The assumptions for PRB size, subframe length, link adaptation constraints, and modulation and coding schemes are aligned with the current LTE-A system. The end user throughput is calculated based on look-up table methods according to the instantaneous received SINR [18], and all the information bits are assumed to be correctly received. Details of the implementations can be found, e.g. in [18]. In the simulation, in total 850 drops are conducted, and each drop contains 40 subframes. New outdoor UEs and MNs are generated in each drop. The ITU-R urban macro cell (UMa) and urban micro cell (UMi) channel models are used for the macro sectors and micro sectors, respectively [12]. The baseline case we compare to is to serve the VUEs directly by the macro cells. Due to the speed of the vehicles, and size of the micro cells, we do not consider serving the VUEs by micro cells in this study. The VUEs are uniformly dropped on each vehicle with $i_{v} \sim \mathcal{U}(1,50)$. The total expected number of users, i.e., macro and micro UEs and VUEs, are kept the same for all the cases.

Figs. 3 and 4 plot the throughput cdf of VUEs when the VPL is at $15 \mathrm{~dB}$ and $30 \mathrm{~dB}$, respectively. From the figures we can see that in both cases, compared to the baseline case, if IRC is used as the backhaul scheme, the throughput of the VUEs are improved significantly. This is as expected, since the system

Table I

SIMULATION PARAMETERS

\begin{tabular}{|c|c|}
\hline Component & Configuration Parameters \\
\hline $\begin{array}{l}\text { Buildings } \\
\text { and Streets }\end{array}$ & $\begin{array}{l}\text { See Fig. } 1.9 \text { buildings } 120 \text { meters by } 120 \text { meters with } 6 \\
\text { floors ( } 3.5 \text { meter height of each floor) } \\
\text { Road width: } 21 \text { meters (sidewalks and parking lanes) }\end{array}$ \\
\hline Macro BS & $\begin{array}{l}\text { Height: } 5 \text { meters above the top of the middle building } \\
\text { (See Fig. } 1 \text { ) } \\
\text { Maximum transmit power (per } 10 \mathrm{MHz} \text { ): } 43 \mathrm{dBm} \\
\text { Carrier: } 800 \mathrm{MHz} \\
\text { Bandwidth: } 20 \mathrm{MHz} \\
\text { Antenna configuration: } 17 \mathrm{dBi}, 3 \text { sectors (one antenna per } \\
\text { sector), } 0,120 \text { and } 240 \text { degrees with respect to the north }\end{array}$ \\
\hline Micro BS & $\begin{array}{l}\text { Height: } 10 \text { meters above the ground close to middle point } \\
\text { of south and east walls } \\
\text { Position: see Fig. } 1 \\
\text { Maximum transmit power (per } 10 \mathrm{MHz} \text { ): } 30 \mathrm{dBm} \\
\text { Carrier: } 2.6 \mathrm{GHz} \\
\text { Bandwidth: } 80 \mathrm{MHz} \\
\text { Cell range expansion bias (CRE): } 5 \mathrm{~dB} \\
\text { Antenna configuration: } 17 \mathrm{dBi} \text { gain, } 2 \text { sectors (one } \\
\text { antenna per sector), pointing to the main street with an } \\
\text { angle of } 20 \text { degrees with respect to the closest wall } \\
\text { ABS duty cycle: } 40 \mathrm{~ms} \text {. }\end{array}$ \\
\hline $\begin{array}{c}\text { Moving } \\
\text { Network }\end{array}$ & $\begin{array}{l}\text { Full-duplex } \\
\text { Speed: } 50 \mathrm{~km} / \mathrm{h} \\
\text { Height: } 3.5 \mathrm{~meters} \text { above the ground } \\
\text { Position: randomly generated according to a Poisson } \\
\text { distribution with } \lambda=0.5 \text { in each direction of the road } \\
\text { Maximum transmit power (per } 10 \mathrm{MHz} \text { ): } 10 \mathrm{dBm} \\
\text { Carrier: } 800 \mathrm{MHz} \text { for backhaul links, and } 2.6 \mathrm{GHz} \text { for } \\
\text { access links } \\
\text { Bandwidth: } 20 \mathrm{MHz} \text { for backhaul links, and } 80 \mathrm{MHz} \text { for } \\
\text { access links } \\
\text { Antenna configuration: single antenna, } 0 \mathrm{dBi} \\
\text { omnidirectional antenna } \\
\text { Receiver noise figure: } 5 \mathrm{~dB}\end{array}$ \\
\hline $\begin{array}{l}\text { Outdoor } \\
\text { UEs } \\
\text { (Macro } \\
\text { UE, Micro } \\
\text { UE) }\end{array}$ & $\begin{array}{l}\text { Speed: } 0 \text { to } 3 \mathrm{~km} / \mathrm{h} \\
\text { Height: } 1.5 \text { meters above the ground } \\
\text { Position: uniformly randomly distributed, } 50 \text { UEs per road } \\
\text { Cell selection: based on received power with } 5 \mathrm{~dB} \text { CRE } \\
\text { bias for micro cells } \\
\text { Receiver noise figure: } 9 \mathrm{~dB}\end{array}$ \\
\hline VUEs & $\begin{array}{l}\text { Height: } 1.5 \mathrm{~m} \text { above the ground } \\
\text { Position: uniformly randomly distributed inside a vehicle } \\
\text { Number of VUEs in each vehicle: uniformly from the } \\
\text { interval }[1,50] \\
\text { Cell selection: always connect to the MN of their own } \\
\text { vehicles } \\
\text { Receiver noise figure: } 9 \mathrm{~dB}\end{array}$ \\
\hline
\end{tabular}




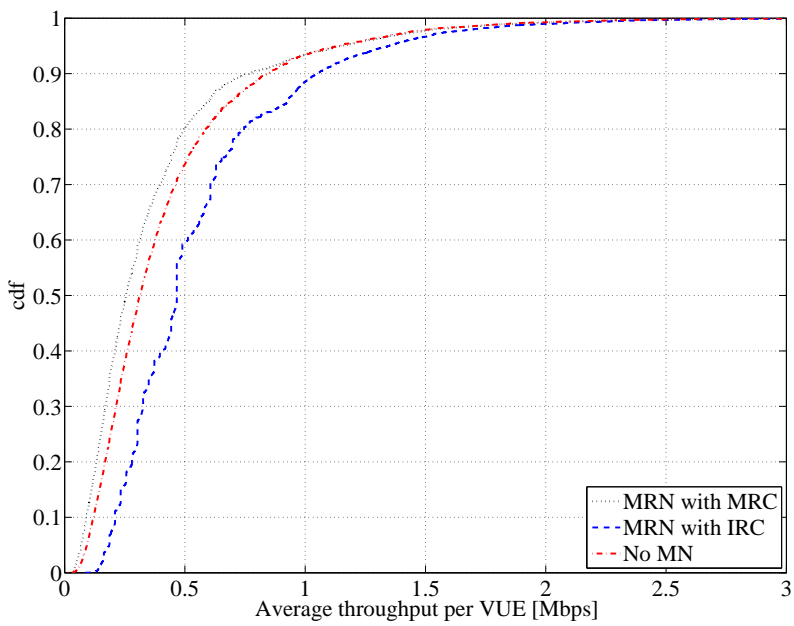

Figure 3. The cdf of VUE throughput, VPL is at $15 \mathrm{~dB}$.

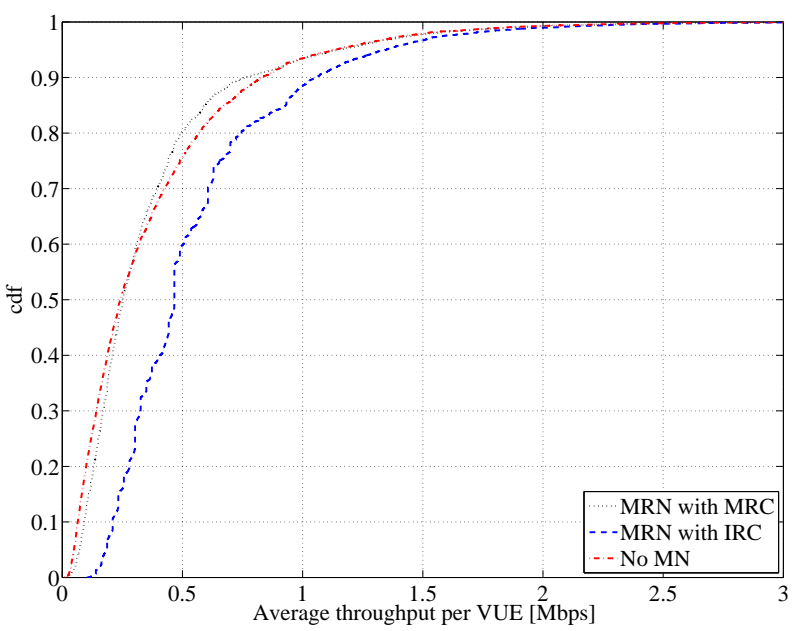

Figure 4. The cdf of VUE throughput, VPL is at $30 \mathrm{~dB}$.

is operating in an interference limited scenario, and IRC can better cope with the interference situation. Regarding the MRC scheme, it only helps the VUEs in the low SINR region when the VPL is high (see Fig. 4). There are two reasons for this: 1. since all the VUEs of a given MN need to be scheduled together, there is less multi-user scheduling gain to use MNs compared to direct transmission; 2. the current LTE-A system requires the same coding and modulation scheme to be used for all the PRBs allocated to a UE during one subframe [13, Chp. 6], which will further limit gains from frequency adaptive scheduling, since the number of PRBs allocated to the backhaul links of an MN is proportional to the number of VUEs it serves. Figs. 5 and 6 plot the throughput cdf of macro UEs when the VPL is at $15 \mathrm{~dB}$ and $30 \mathrm{~dB}$, respectively. As we can see from the figures that after introducing MNs to the system, compared to the baseline case, there is no obvious change of the throughput of macro UEs.

Figs. 7 and 8 plot the throughput cdf of micro UEs when the VPL is at $15 \mathrm{~dB}$ and $30 \mathrm{~dB}$, respectively. As ABSs are configured for micro cells, the degradation of the micro UEs throughput is expected. The degradation is more obvious when the VPL is low, and when the backhaul link has higher

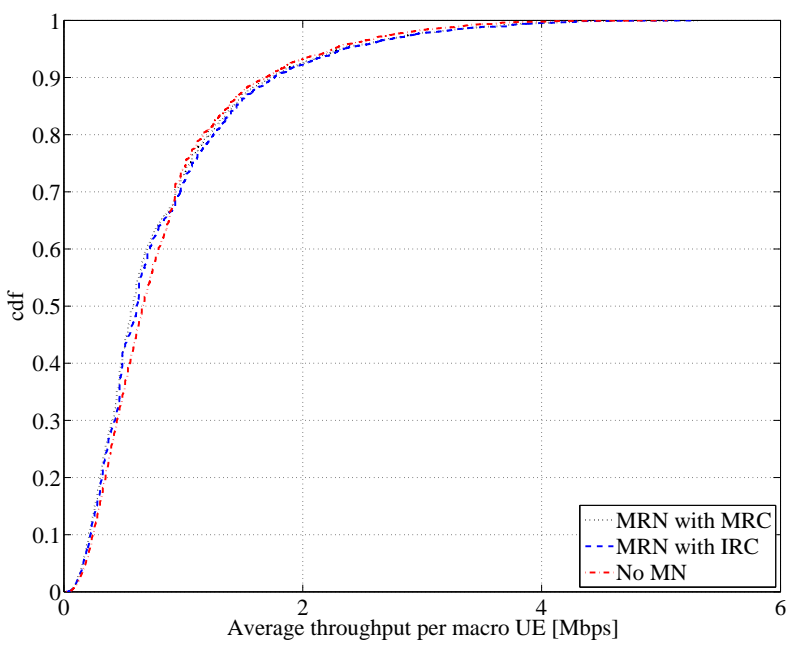

Figure 5. The cdf of macro UE throughput, VPL is at $15 \mathrm{~dB}$.

throughput (see Fig. 7). This is because more ABSs needs to be configured by the macro cells to protect the access links of the MNs, and the interference from the access links of MNs also impact the nearby micro UEs. However, when the VPL is $30 \mathrm{~dB}$, the degradation of micro UEs is less obvious, and there is no difference between the IRC and MRC backhaul schemes. This is because when the VPL is high, the vehicle itself can attenuate the interference significantly, and less interference is leaked out from the vehicle to the nearby micro UEs.

\section{Discussion AND CONCLUSIONS}

The use of MNs can improve the performance of the VUEs in ultra-dense urban scenarios, if advanced receivers are used. This is one of the biggest advantages of using MNs as they are less constrained by antenna space, power and transceiver complexities. Moreover, the impact of using MNs on regular outdoor users are very limited. However, the backhaul links are still the bottle necks to further improve the performance of MNs. The performance of backhaul links can be further improved in several ways, especially in a densely deployed scenario. For example, only up to 64-QAM is supported in the current LTE-A system as the modulation methods. In a densely deployed scenario, the SINR can support the use of 256-QAM [19]. Moreover, as demonstrated in [6], by using advanced antenna systems on public transportation systems, reliable CSI can be obtained at the transmitter side which enables the use of more advanced multi-antenna schemes. Certainly modifications of the current mobile communication systems are required to support MNs. This issue has been discussed in [4], [7]. However, most of the components at the network side can be reused, and only new protocols need to be introduced to support the mobility of the MNs. The cost of deploying MNs on the public transportation vehicles can be recouped by bringing in more business opportunities for operators and service providers. Nevertheless, the use of MNs is very promising for the next generations of mobile communication systems to improve the experiences of VUEs. 


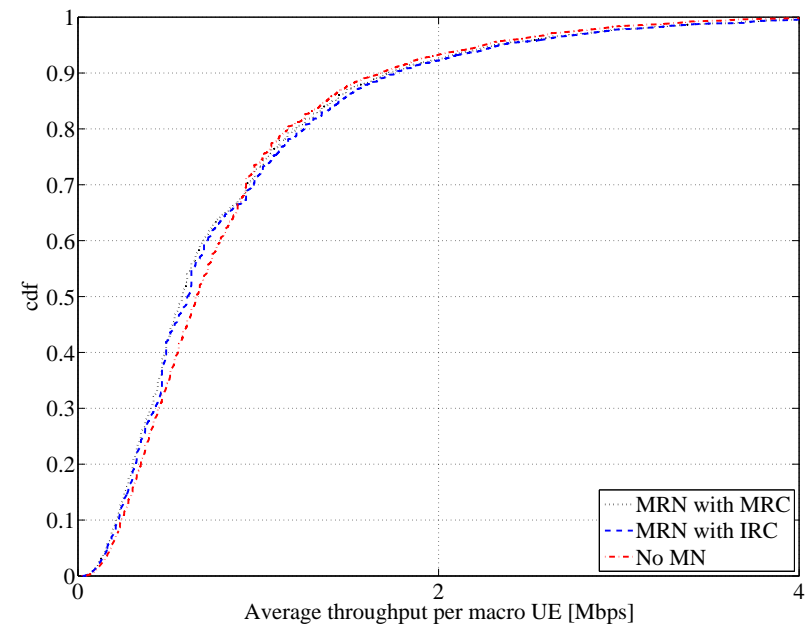

Figure 6. The cdf of macro UE throughput, VPL is at $30 \mathrm{~dB}$

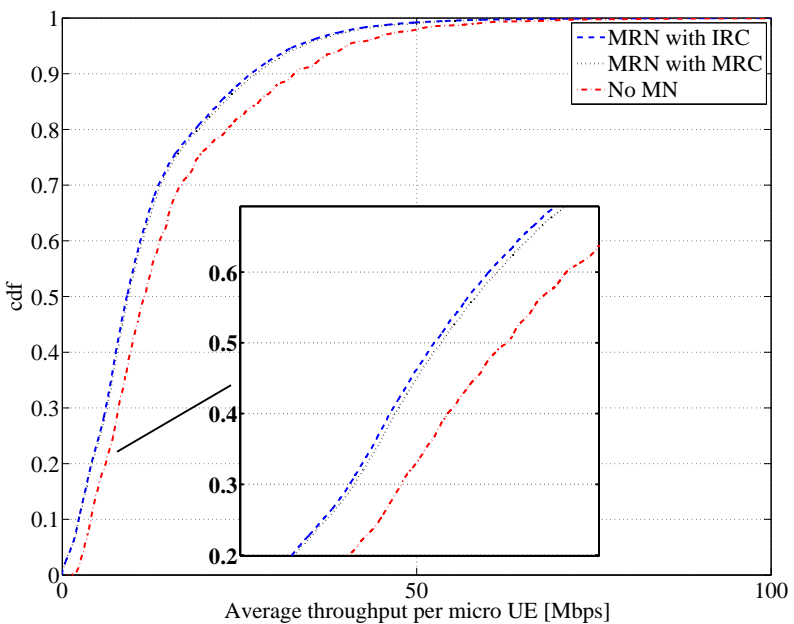

Figure 7. The cdf of micro UE throughput, VPL is at $15 \mathrm{~dB}$.

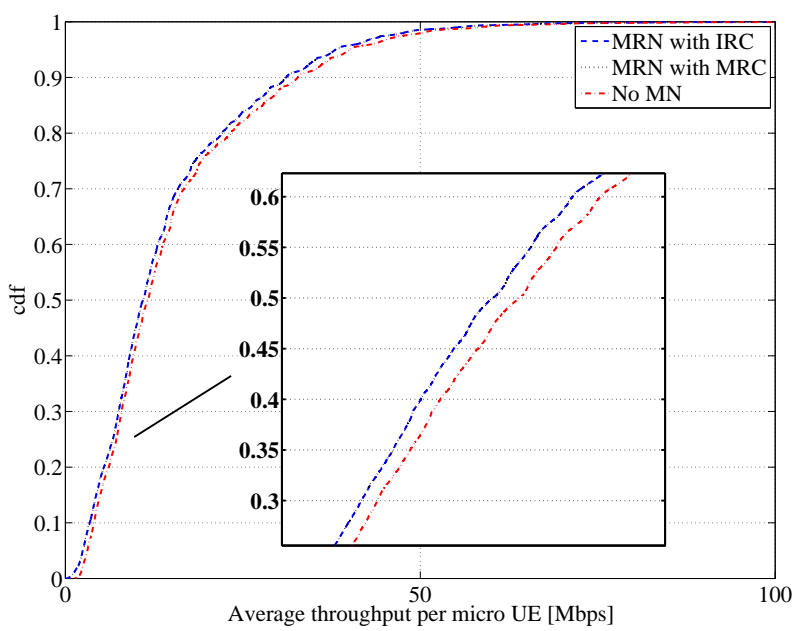

Figure 8. The cdf of micro UE throughput, VPL is at $30 \mathrm{~dB}$.

\section{ACKNOWLEDGMENT}

Part of this work has been performed in the framework of the FP7 project ICT-317669 METIS, which is partly funded by the European Union. The authors would like to acknowledge the contributions of their colleagues in METIS, although the views expressed are those of the authors and do not necessarily represent the project. This work is also supported by the Swedish Research Council VR under the project 621-20094555 Dynamic Multipoint Wireless Transmission.

\section{REFERENCES}

[1] I. Hwang, B. Song, and S. S. Soliman, "A holistic view on hyper-dense heterogeneous and small cell networks," IEEE Commun. Mag., vol. 51, pp. 20-27, Jun. 2013.

[2] I. S. Gaspar and G. Wunder, "5G Cellular communications scenarios and system requirements," Tech. Rep., [Online, last accessed 2014-1001] https://www.5gnow.eu.

[3] P. Popovski, V. Braun, H.-P. Mayer, P. Fertl et al., "ICT-317669METIS/ D1.1 Scenarios, requirements and KPIs for 5G mobile and wireless system," Tech. Rep., [Online, last accessed 2014-10-01] https://www.metis2020.com.

[4] 3GPP TR 36.836, "Technical Specification Group Radio Access Network; Mobile Relay for Evolved Universal Terrestrial Radio Access (E-UTRA)," Tech. Rep., [Online, last accessed 2014-10-01] http://www.3gpp.org.

[5] Y. Sui, J. Vihrälä, A. Papadogiannis, M. Sternad et al., "Moving cells: a promising solution to boost performance for vehicular users," IEEE Commun. Mag., vol. 51, pp. 62-68, Jun. 2013.

[6] D.-T. Phan-Huy, M. Sternad, and T. Svensson, "Adaptive large MISO downlink with predictor antennas array for very fast moving vehicles," in Proc. IEEE Int. Conf. on Connected Veh. and Expo (ICCVE), 2013.

[7] A. Saadani, G. Liebl et al., "D3.4 relay configurations," Tech. Rep., Artist4G deliverable, Jul. 2011, [Online, last accessed 2014-10-01] https://ict-artist4g.eu/projet/deliverables.

[8] E. Tanghe, W. Joseph, L. Verloock, and L. Martens, "Evaluation of vehicle penetration loss at wireless communication frequencies," IEEE Trans. Veh. Technol., vol. 57, no. 4, pp. 2036-2041, Jul. 2008.

[9] Y. Sui, A. Papadogiannis, and T. Svensson, "The potential of moving relays-a performance analysis," in Proc. IEEE Conf. Veh. Tech. (VTC), 2012-Spring.

[10] Y. Sui, Z. Ren, W. Sun, T. Svensson et al., "Performance study of fixed and moving relays for vehicular users with multi-cell handover under co-channel interference," in Proc. IEEE Int. Conf. on Connected Veh. and Expo (ICCVE), 2013.

[11] W. Li, C. Zhang, X. Duan, S. Jia et al., "Performance evaluation and analysis on group mobility of mobile relay for LTE-Advanced system," in Proc. IEEE Conf. Veh. Tech. (VTC), 2012-Fall.

[12] P. Agyapong, V. Braun, M. Fallgren, A. Gouraud et al., "ICT-317669METIS/D6.1 Simulation guidelines," Tech. Rep., 2013, [Online, last accessed 2014-10-01] https://www.metis2020.com.

[13] S. Sesia, I. Toufik, and M. Baker, LTE - The UMTS Long Term Evolution: From Theory to Practice, 2nd ed. West Sussex, UK: John Wiley \& Sons Ltd., 2011.

[14] F. Kelly, A. Maulloo, and D. Tan, "Rate control for communication networks: shadow prices, proportional fairness and stability," Journal of the Operational Research Society, vol. 49, pp. 237-252, Apr. 1998.

[15] D. G. Brennan, "Linear diversity combining techniques," Proceedings of the IEEE, vol. 47, pp. 1075-1102, Jun. 1959.

[16] J. Zhang, J. Olivier, A. Sayeed, and B. V. Veen, "Low complexity MIMO receiver via maximum SINR interference cancellation," in Proc. IEEE Conf. Veh. Tech. (VTC), 2002-Spring.

[17] M. Simsek, M. Bennis, and A. Czylwiki, "Dynamic inter-cell interference coordination in HetNets: A reinforcement learning approach," in Proc. IEEE Global Commun. Conf. (GLOBECOM), 2012.

[18] G. Piro, L. A. Grieco, G. Boggia, F. Capozzi et al., "Simulating LTE cellular systems: an open source framework," IEEE Trans. Veh. Technol., vol. 60, pp. 498-513, Nov. 2011.

[19] 3GPP TR 36.872, "Small cell enhancements for E-UTRA and EUTRAN - physical layer aspects," Tech. Rep., 2013, [Online, last accessed 2014-10-01] http://www.3gpp.org. 